Relations industrielles

Industrial Relations

\title{
Le harcèlement sexuel au travail: définition et mesure du phénomène
}

\section{Dominique Savoie et Viateur Larouche}

Volume 43, numéro 3, 1988

URI : https://id.erudit.org/iderudit/050430ar

DOI : https://doi.org/10.7202/050430ar

Aller au sommaire du numéro

Éditeur(s)

Département des relations industrielles de l'Université Laval

ISSN

0034-379X (imprimé)

1703-8138 (numérique)

Découvrir la revue

\section{Citer cet article}

Savoie, D. \& Larouche, V. (1988). Le harcèlement sexuel au travail: définition et mesure du phénomène. Relations industrielles / Industrial Relations, 43(3),

509-530. https://doi.org/10.7202/050430ar
Résumé de l'article

Après avoir défini ce qu'il faut entendre par harcèlement sexuel au travail, les auteurs proposent une grille d'identification permettant de classifier selon la forme et le degré les différents comportements qui caractérisent le harcèlement sexuel au travail.
Tous droits réservés (C) Département des relations industrielles de l'Université Laval, 1988
Ce document est protégé par la loi sur le droit d'auteur. L'utilisation des services d'Érudit (y compris la reproduction) est assujettie à sa politique d'utilisation que vous pouvez consulter en ligne.

https://apropos.erudit.org/fr/usagers/politique-dutilisation/ 


\title{
Le harcèlement sexuel au travail
}

\section{Définition et mesure du phénomène}

\author{
Dominique Savoie \\ et \\ Viateur Larouche
}

Après avoir défini ce qu'il faut entendre par harcèlement sexuel au travail, les auteurs proposent une grille d'identification permettant de classifier selon la forme et le degré les différents comportements qui caractérisent le harcèlement sexuel au travail.

L'avènement de la société industrielle a suscité de nombreux bouleversements qui n'ont pas été sans créer des problèmes d'importance. Un de ces problèmes a été l'éclatement de la cellule familiale comme lieu de travail. Ainsi la création des premières «manufactures» en Angleterre, a fait que hommes, femmes et enfants ont dû, dès lors, délaisser leur foyer pour se retrouver dans des milieux communs de travail afin d'y gagner la rémunération nécessaire pour vivre.

Dans un tel contexte, on note que les femmes sont apparues plus tardivement que les hommes dans ces différents milieux de travail quoique leur venue $n$ 'a fait que croître ${ }^{1}$ depuis la fin de la dernière guerre mondiale. L'arrivée de cette main-d'oeuvre ne s'est pas faite sans créer de nombreux problèmes de discrimination sexiste au travail. Les attitudes discriminatoires véhiculées par la société industrielle patriarcale ont rendu difficile, pour les femmes, l'accès au marché du travail et ont permis l'élaboration et le maintien de conditions de travail différentes pour elles comparativement à celles

- SAVOIE, D., étudiante au doctorat, Département des relations industrielles, Université Laval

L.AROUCHE, V., professeur, Ecole de relations industrielles, Université de Montréal

** Pour la préparation et la diffusion de cette étude, les auteurs ont bénéficié d'une subvention du Conseil québécois de la recherche en sciences sociales et du Fonds FCAR.

1 Pat ARMSTRONG and Hugh ARMSTRONG, $A$ Working Majority: What Women Must Do For Pay, 1983, p. 7, 249. En 1946, un quart des femmes canadiennes travaillait ou cherchait un emploi. En 1966, un tiers des femmes était sur le marché du travail. 
des hommes. Encore aujourd'hui, malgré un effort sérieux de la part du législateur ${ }^{2}$ pour faire sanctionner des lois contre la discrimination (selon le sexe) dans l'emploi, il existe encore des formes subtiles de discrimination à l'égard des femmes telle que le harcèlement sexuel au travail.

À cet égard, les femmes se sont tues pendant bien longtemps et ce n'est que depuis un peu plus de dix ans qu'elles expriment de plus en plus l'existence de ce problème ${ }^{3}$.

Néanmoins, une des premières difficultés qu'il faille surmonter face à ce phénomène, est celle d'en arriver à le définir d'une façon complète et réaliste et qui se prête à la mesure.

L'objectif du présent article est double. Dans un premier temps, les auteurs se proposent de définir ce qu'il faut entendre par harcèlement sexuel au travail. Dans cette ligne de pensée, ils débouchent sur une définition conceptuelle et opérationnelle du phénomène.

Dans un second temps, les auteurs proposent une grille d'identification permettant de classifier selon la forme et le degré les différents comportements qui caractérisent le harcèlement sexuel au travail.

\section{DÉFINITION DU HARCÈLEMENT SEXUEL}

Les définitions du harcèlement sexuel au travail qui apparaissent dans la littérature sont issues principalement soit du milieu juridique ou du milieu

2 Le législateur québécois et canadien pour ne mentionner que ces deux (2) palliers de gouvernement, par la Charte des droits et libertés de la personne, L.R.Q., c. C-12, ou la Loi canadienne sur les droits de la personne, S.C. 1976-1977, c. 33.

3 Constance BACHOUSE and Leah COHEN, Sexual Harassment on the Job: How to Avoid the Working Woman's Nightmare, 1981, p. 40; Commission canadienne des droits de la personne, Attentions sexuelles non sollicitées et harcèlement sexuel: résultats d'un sondage auprès des canadiens, 1983; Lise MOISAN, «Les dessous du 9 à 5: suite et fin?", la Vie en Rose, septembre-octobre 1982, p. 17. En 1976, aux Etats-Unis, la revue Redbook publie les résultats d'un questionnaire qui démontre que $88 \%$ des 9,000 répondantes avaient subi une forme ou une autre de harcèlement sexuel et que $92 \%$ d'entre elles le considéraient comme un problème grave. Au Canada, selon les résultats d'un sondage effectué en septembre 1981 par la maison CROP de Montréal pour le compte de la Commission canadienne des droits de la personne, $49 \%$ des femmes et $33 \%$ des hommes ayant répondu à ce sondage ont dit avoir subi des attentions sexuelles non sollicitées. Cependant, après avoir vérifié auprès d'eux s'ils considéraient ces attentions comme étant du harcèlement sexuel, le rapport conclut que... $\ll 15 \%$ des Canadiennes et $4 \%$ des Canadiens ont à un moment donné de leur vie fait l'objet d'attentions sexuelles importunes qu'ils ont considérées comme du harcèlement sexuel». Au Québec, dans un sondage effectué par la revue $L a$ Vie en Rose, $64 \%$ des 2,465 répondantes déclarent avoir été harcelées sexuellement. 
féministe. Dans certains cas, les auteurs ont privilégié une démarche conceptuelle alors que dans d'autres cas, ils ont préféré une analyse plus descriptive.

À la lecture de la jurisprudence canadienne, il ne semble pas que les tribunaux aient eu de grandes difficultés à en reconnaître la présence ${ }^{4}$. En effet, les tribunaux reconnaissent que le harcèlement sexuel peut être fondamentalement de deux types, soit celui qui conduit à des conséquences négatives dans les conditions de travail et celui qui empoisonne un environnement de travails.

Il faut préciser que, dans les faits, le problème de la définition du harcèlement sexuel s'est peu posé parce qu'à l'analyse de chaque cas, les faits reprochés au harceleur étaient suffisamment clairs (forme physique) pour conclure qu'il s'agissait de harcèlement sexuel. Lorsque le harcèlement prenait une forme verbale, celle-ci était suffisamment explicite pour qu'encore une fois la présence de harcèlement sexuel soit reconnue.

Néanmoins, les tribunaux se sont quand même penchés sur le problème de la définition du harcèlement sexuel. On peut dire que, généralement, ils ont retenu la définition établie par $\mathrm{O}$. B. Shime dans la première cause de harcèlement sexuel à être entendue au Canada.

"The forms of prohibited conduct that, in my view, are discriminatory run the gamut from overt gender-based activity, such as coerced intercourse to unsolicited physical contact to persistent propositions to more subtle conduct such as genderbased insults and taunting, which may reasonably be perceived to create a negative psychological and emotional work environment. $\nu^{6}$

Il faut souligner que la Commission canadienne des droits de la personne a aussi, suite à quelques décisions, établi une définition du harcèlement sexuel. Cette définition se lit comme suit:

Pour être qualifiée de harcèlement sexuel, toute action doit répondre à l'un des critères suivants: être perçue à juste titre comme une condition d'emploi...; influer sur les décisions prises en ce domaine, nuire au rendement professionnel... ou humilier, insulter ou intimider la personne qui subit cette action. Est considérée comme du harcèlement sexuel toute action dont l'importunité ne pouvait échapper à son auteur». ${ }^{7}$

4 Le principal problème des tribunaux, lorsqu'ils rejettent une plainte, n'en est pas un de définition du harcèlement sexuel mais plutôt un problème de preuve.

5 Pour une meilleure compréhension de ces deux types de harcèlement, lire l'ouvrage suivant: Catharine A. MACKINNON, Sexual Harassment of Working Women: A Case of Sex Discrimination, 1979, 312 pp.

6 Bell and Korczak v. Ernest Ladas and the Flaming Steer Steak House, 1 C.H.R.R., D/155, D/159, le 12 aout 1980, O.B. Shime.

7 Commission canadienne des droits de la personne, op. cit., p. 5. 
Bref, l'analyse juridique permet de faire ressortir quelques éléments qu'il apparaît intéressant de souligner. La nature des comportements subis doit évidemment être à connotation sexuelle. Il faut de plus souligner que l'emphase est souvent mise sur les conséquences vécues par la victime.

Notons que la répétition de ces comportements ne semble pas nécessaire pour juger que ceux-ci constituent du harcèlement sexuel. Selon O.B. Shime, «un seul incident où un employé se voit nier l'égalité dans l'emploi à cause de son sexe est aussi prohibé par la loi» ${ }^{8}$. Par la suite, d'autres ont raffiné cette affirmation en précisant que, dans certains cas, un comportement non répété pouvait constituer du harcèlement, lorsqu'il en résulte la perte d'un avantage, lorsqu'il y a assaut ou lorsqu'une proposition est tellement grossière ou obscène que l'environnement de travail d'une victime devient empoisonné. Dans les autres cas, la répétition fait en sorte qu'un comportement qui, se produisant une seule fois, ne serait pas condamnable, le devient $^{9}$. La notion de répétition des comportements est donc généralement soulevée lorsque le harcèlement prend une forme verbale.

Enfin, le rejet par la victime de ces comportements est, sinon explicite, du moins suffisamment implicite pour le considérer comme un élément important de la définition.

Quant à l'analyse féministe, elle s'appuie plutôt sur les relations de pouvoir (historiques) entre les hommes et les femmes ainsi que sur les diverses perceptions que chacun d'eux ont pu établir en ce qui concerne, entre autres, la place de la femme sur le marché du travail ainsi que la sexualité. Deux définitions de ce genre retiennent particulièrement notre attention.

«Sexual harassment is described as unsolicited non reciprocal male behavior that asserts a woman's sex role over her function as worker.» ${ }^{10}$

«Sexual harassment involves the intimidation of women by men who use their positions of power and authority over women in order to exact sexual favors that would not otherwise be granted.» 11

D'autres groupes, tant aux États-Unis qu'au Canada, s'inscrivent aussi dans le courant féministe bien que leur définition revête un caractère nettement plus descriptif. Tel est le cas du comité d'action contre le harcèlement sexuel du groupe populaire Au Bas de l'Échelle qui le définit comme suit:

8 Bell and Korczak v. Ernest Ladas and the Flaming Steer Steak House, op. cit., (traduction libre).

9 Kotyk and Allary v. Canadian Employment and Immigration Commission and Jack Chuba, 4 C.H.R.R. D/1416, D/1430, le 20 avril 1983, Susan M. Ashley.

10 Lin FARLEY, Sexual Shakedown: The Sexual Harassment of Women on the Job, 1978, p. 33.

11 John C. HUGHES and Larry MAY, "Sexual Harassment», Social theory and Practice, Vol. 6, No. 3, Fall 1980, p. 249. 
«... toutes formes d'avances et de demandes à connotation sexuelle, non-désirées, comprenant des gestes, des paroles, des attouchements, des invitations, des propositions, etc., allant jusqu'à son expression la plus grossière telle que l'assaut physique et le viol. De plus, le harcèlement sexuel comprend une connotation d'agressivité, de domination et de répétition, bien que la répétition ne soit pas nécessaire pour causer des dommages à une victime. Bien souvent, le harcèlement est accompagné de menaces directes ou sous-entendues de représailles (telles que refus d'embauche, congédiement, détérioration de conditions de travail, mauvaises références, etc.) ou rend la situation au travail intolérable, obligeant une victime à demander un transfert ou à démissionner de son emploi.» ${ }^{12}$

Quant au mouvement syndical, en général, son action s'est inscrite dans le contexte de ce mouvement. Ses définitions sont parfois asexuées, identifiant clairement les victimes comme étant de sexe féminin. Cependant, tout le matériel didactique utilisé en vue de conscientiser les membres à ce problème identifie la femme comme étant la victime visée presqu'exclusivement par ce problème.

«Toute pratique orientée vers la sexualité qui met en péril l'emploi d'une femme, nuit à son rendement de travail et menace sa productivité économique fait partie de ce que l'on appelle le harcèlement sexuel au travail. C'est aussi un commentaire sexuel et non désiré, un contact suggestif ou physique qu'une femme considère comme répréhensible, désagréable ou offensant qui l'incommode dans son travail.» ${ }^{13}$

Il est clair que l'analyse féministe s'intéresse plus particulièrement au harcèlement sexuel au travail vécu par les femmes et exercé par les hommes. Bien que bon nombre d'études démontrent que la très grande majorité des personnes harcelées sont des femmes, on ne peut nier que, dans certains cas, plus rares, les hommes sont aussi harcelés ${ }^{14}$.

L'élément principal qui caractérise les définitions issues de la démarche féministe est la notion de pouvoir. De plus, cette analyse, comme celle qui est à caractère juridique, accorde peu d'importance au critère de répétition des comportements. Elle s'intéresse aux conséquences vécues par les victimes sans pour autant ignorer les comportements à proscrire.

Bref, les analyses juridiques et féministes permettent de dégager certains critères utiles pour la formulation d'une définition du harcèlement sexuel. Il faut cependant noter que le consensus est loin d'être établi puisque de nombreux auteurs et organismes utilisent encore une approche très des-

12 Comité d'action sur le harcèlement sexuel, "Questionnaire sur le harcèlement sexuel», Au Bas de l'Échelle, Circa 1982, p. 1 (non publiè).

13 Fédération des travailleurs du Québec, Le harcèlement sexuel, C'est quoi?, Montréal, FTQ, 1983, pp. 9-10; il s'agit d'une définition soumise par le Syndicat canadien de la fonction publique.

14 Le problème des hommes victimes de harcèlement sexuel est discuté plus loin dans le présent article. 
criptive du problème. Dans le but d'articuler une définition claire et concise du harcèlement sexuel au travail, il importe donc d'analyser chacune des composantes de ce phénomène.

\section{Le concept de harcèlement}

Hughes et May se sont intéressés à l'étude de la notion de harcèlement. Selon eux, on peut définir le harcèlement comme suit:

«Harassment refers to a class of annoying or unwelcome acts undertaken by one person (or group of persons) against another person (or group of persons). The purpose of this act may be merely to annoy, but more frequently the aim is to coerce another person into doing something he or she would not normally choose to do. $\rangle^{15}$ (Italiques des auteurs)

Le dictionnaire Larousse définit aussi «harceler» comme étant «fatiguer par des attaques répétées; importuner, agacer: il le harcèle sans cesse de demandes, de questions» ${ }^{16}$.

Ces deux définitions permettent de déceler deux dimensions, soit la notion de répétition des actions posées et le but recherché par ces actions, qu'il s'agisse de contrarier une personne ou de la contraindre. La notion de répétition des comportements harcelants revêt un caractère important même si elle est très peu retenue dans les diverses définitions que nous retrouvons dans la littérature. Une certaine tendance se dégage pourtant au niveau opérationnel ${ }^{17}$ pour en faire un critère presque toujours présent sauf dans les cas les plus sérieux où l'on retrouve des menaces très précises, des assauts ou des viols ${ }^{18}$.

«Quoiqu'il soit vrai de dire que plus l'approche est subtile, plus elle doit être répétée souvent afin d'en déterminer correctement la nature, il est aussi vrai de dire, inversement, que plus l'approche est évidente et grossière (i.e. dommageable), moins elle doit être répétée souvent avant qu'en soit déterminée correctement la nature.»19

Il faut cependant souligner que Hughes et May considèrent qu'un seul acte, même non répété, peut être considéré comme du harcèlement puisqu'il fait partie de l'ensemble des actes posés quotidiennement dans le but de dominer les femmes ${ }^{20}$. Cette approche est intéressante lorsqu'on l'applique

15 HUGHES and MAY, op. cit., p. 250.

16 Petit Larousse, 1959, p. 501.

17 En effet, la majorité des questionnaires que nous avons pu consulter contenaient tous des questions directes ou indirectes se rapportant à la répétition des comportements. Voir, entre autres, Comité d'action sur le harcèlement sexuel, 1982, op. cit., pp. 3-4.

18 Jennifer JAMES, «Sexual Harassment», Public Personnel Management, Vol. 10, No. 4, Winter 1981, p. 403.

19 André DAGILIS et Elisabeth NOVAK, Le harcèlement sexuel? Connais pas..., 1982, p. 11.

20 HUGHES and MAY, op. cit., p. 251. 
à certains milieux de travail où le même geste harcelant peut être posé par plusieurs personnes, chacune d'elles ne le répétant pourtant pas. On pourrait alors parler de milieux de travail harcelants sans pouvoir identifier un harceleur en particulier ${ }^{21}$.

Donc, sans rejeter l'approche de Hughes et de May, il faut néanmoins considérer qu'un critère de répétition des comportements harcelants est valable lorsque nuancé selon la forme de comportement observé et le but recherché.

En analysant les buts recherchés par un harceleur, les auteurs précités affirment que le harcèlement sexuel est véritablement du harcèlement. Ils distinguent trois types de situations qui caractérisent le harcèlement sexuel, soit des situations contrariantes (par exemple, remarques sexuelles, se faire continuellement déshabiller des yeux), des situations de menaces (par exemple, si tu ne fais pas «x», je vais te congédier) et des situations de promesses ou d'offres (par exemple, si tu fais « $\mathrm{x} »$, tu auras une promotion) ${ }^{22}$. La première et la deuxième situations rejoignent clairement les buts recherchés par le harcèlement, soit, dans le premier cas, de contrarier une personne et dans le second cas, de la placer dans une situation contraignante.

«If someone explicitly proposes to make another worse off than before, were this person not to comply with a demand or request, then he or she has been threatened, and because of this coerced, and perhaps because of this, harmed. ${ }^{23}$

Cette constatation est cependant moins claire dans le cas de la troisième situation proposée qui en est une d'offres. Michael Bayles croit, en effet, qu'une telle situation n'a aucun effet de contrainte puisque la personne qui rejette l'offre qui lui a été faite ne se retrouve pas en moins bonne position qu'auparavant alors qu'en l'acceptant, elle se retrouve en meilleure position qu'auparavant. Donc, cette personne ne fait face à aucun choix qui pourrait la désavantager ${ }^{24}$. Cette conclusion est difficilement acceptable pour plusieurs motifs. Dans un premier temps, il existe une catégorie d'offres qui rend le rejet difficile. Tel semble être le cas lorsque c'est un supérieur qui formule l'offre. N'est-il pas vrai que celui qui a la possibilité de faire l'offre (de donner une promotion, par exemple) a aussi la possibilité de retirer les avantages qui y sont associés en cas de refus? Dans un second temps, l'argument de Bayles doit être réfuté parce que suite à l'acceptation d'une telle of-

21 L'exemple typique de ces milieux de travail harcelants pourrait être celui d'un restaurant où la même serveuse peut subir, dans une même journée, les blagues et les gestes importuns de nombreux clients.

22 HUGHES and MAY, loc. cit.

23 Ibid., p. 252.

24 Michael BAYLES, «Coercive Offers and Public Benefits», The Personalist, Vol. 55, Spring 1974, pp. 142-143. 
fre, ladite personne risquera de se trouver en position affaiblie. Qui ne connaît pas la nature changeante des sentiments d'ordre sexuel? De plus, la personne qui a accepté une telle offre sera plus vulnérable, la personne lui ayant fait l'offre la tiendra en son pouvoir. Enfin, si une telle exigence se généralise, le pouvoir qui a été exercé, à un moment donné, sous forme d'offres, sera peut-être exercé, dans le futur, sous forme de menaces ${ }^{25}$.

«Sexual offers as well as sexual threats are coercive because they present the victim with new undesirable consequences of her actions. ${ }^{26}$

Nous pouvons donc conclure que le harcèlement sexuel au travail recoupe la définition du harcèlement lorsqu'un critère nuancé de répétition des comportements est présent et lorsque le but ou l'effet ${ }^{27}$ de ces comportements consistent à contrarier une personne ou à la contraindre à faire quelque chose qu'elle ne ferait pas sans cette contrainte.

\section{Le concept de sexualité}

La très grande majorité des auteurs qui se sont intéressés à ce sujet, s'entendent, aujourd'hui, pour reconnaître que le processus de socialisation (l'éducation) des hommes et des femmes est en grande partie responsable de leurs perceptions respectives des rôles sexuels de chacun des groupes ${ }^{28}$. Les stéréotypes dans lesquels chacun des deux sexes est encadré remontent très loin dans le temps et perpétuent l'image de l'homme conquérant, de la

25 HUGHES and MAY, op. cit., pp. 255-256.

26 Ibid., p. 256.

27 Nous ajoutons la notion d'effets à celle de buts, tel que la présente l'évolution de la preuve en matière de discrimination, puisqu'il serait trop facile pour un harceleur de se défendre en disant qu'il ne visait aucun des buts qui définissent le harcèlement alors que les effets y sont. Walter Surma TARNOPOLSKY, Discrimination and the Law in Canada, 1982, p. 275.

28 Les personnes intéressées à une meilleure compréhension du rôle de l'éducation dans la perception des rôles sexuels, selon diverses orientations, peuvent lire les ouvrages suivants: Elena GIANINI BELOTTI, Du côté des petites filles, 1974, 251 pp.; Margaret MEAD, Moeurs et sexualité en Océanie, 1971, 533 pp.; Christiane OLIVIER, Les enfants de Jocaste, 1980, 192 pp.; Michaels KORDA, Male Chauvinism! How it Works, 1972, 244 pp.; Connie BACHOUSE et al., Fighting Sexual Harassment: An Advisory Handbook, 1979, 92 pp.; Mary COELI MEYER et al., Sexual Harassment, 1981, 200 pp.; Mariette LEVESQUE, Bas les pattes! Le Harcèlement sexuel, 1982, 252 pp. 
femme passive et soumise, l'image de l'homme-sujet et de la femme-objet, tout en instaurant des normes sexuelles plus rigides pour les femmes que pour les hommes ${ }^{29}$.

Les hommes ont donc, depuis longtemps, utilisé la sexualité comme moyen de domination envers les femmes tel que le souligne Susan Brownmiller lorsqu'elle étudie le phénomène du viol dans les sociétés primitives.

«Les études ethnologiques des peuples primitifs fort éloignés de nous montrent que le viol est considéré comme une expression de virilité, comme une marque du concept de propriété appliqué aux femmes, et comme un mécanisme de contrôle social destiné à maintenir les femmes dans le rang. Il n'en a pas été différemment dans d'autres parties du monde, sinon de fait, du moins souvent, dans les fantasmes privés et publics des hommes qui dominent et définissent la culture. $)^{30}$

L'équation entre le pouvoir et la sexualité masculine est encore très présente, aujourd'hui, tant dans la mentalité des hommes que dans celle des femmes.

Dans la vie de tous les jours, les femmes sont soumises aux contrariétés et contraintes issues de ces perceptions des rôles sexuels. En effet, le harcèlement sexuel quotidien est vécu par bon nombre de femmes, parfois dans ses expressions les plus contrariantes telles se faire siffler sur la rue, être confrontée à l'étalage pornographique à la télévision, dans les cinémas ou chez les marchands de journaux ou se faire "achaler» régulièrement dans les bars, les restaurants ou les cinémas. Néanmoins, il semblerait que beaucoup de femmes vivent ce harcèlement sexuel quotidien dans des expressions

29 Les personnes intéressées par une analyse du développement de ces stéréotypes et de leur impact dans les cas de harcèlement sexuel au travail, peuvent lire les ouvrages et articles suivants: MEYER et al., op. cit., pp. 49-71; BACHOUSE and COHEN, op. cit., p. 38; George E. BILES, «A Program Guide For Preventing Sexual Harassment in the Workplace», The Personnel Administrator, Vol. 26, No. 6, June 1981, pp. 49-56; Lucie LAURIN et Johanne VOGHEL, Viol et brutalité, 1983, p. 145; Caroll M. BRODSKY, «Rape at Work», in: Marcia J. Walker, Stanley L. Brodsky (editors), Sexual Assault: The Victim and the Rapist, 1976, p. 51; Susan H. KLEMMACK and David L. KLENMACK, "The Social Definition of Rape", in: Ibid., p. 136; James C. RENICK, «Sexual Harassment at Work: Why it Happens, What to Do About It», Personnel Journal, Vol. 59, No. 8, August 1980, p. 659; David L. BRADFORD et al., "The Executive Man and Woman: The Issue of Sexuality», in: D.A. Neugarten and J.M. Shafritz (editors), Sexuality in Organizations: Romantic and Coercive Behaviors at Work, 1980, pp. 17-28.

30 Susan BROWNMILLER, Le Viol, 1976, p. 349. Elle s'appuie principalement sur les études ethnologiques suivantes: Herbert J. ROSE, A Handbook of Greek Mythology, 1928, 363 pp.; Margaret MEAD, Sex and Temperament in Three Primitive Societies (third edition), 1963, 335 pp.; Margaret MEAD, Male and Female, 1949, 477 pp.; Robert F. MURPHY, «Social Structure and Sex Antagonism», Southwestern Journal of Anthropology, Vol. 15, 1959, pp. 89-98. 
beaucoup plus contraignantes: les abus sexuels ou l'inceste chez la jeune enfant, ${ }^{31}$ les avances des professeurs chez la jeune adolescente, ${ }^{32}$ ou la peur quotidienne du viol, ${ }^{33}$ «acte de pression et de colère» ${ }^{34}$ avant d'être un acte sexuel.

Le concept de pouvoir dans le milieu de travail

Le cadre d'analyse qui peut le mieux nous permettre jusqu'ici de cerner une définition conceptuelle du harcèlement sexuel au travail est celui des féministes pour lesquelles le coeur du problème se situe dans les rapports de pouvoir entre les hommes et les femmes. Néanmoins, si cette analyse cerne bien la dimension des rapports harceleurs-hommes et harcelées-femmes, elle laisse quelque peu dans l'ombre les rapports femmes-hommes, hommeshommes et femmes-femmes ${ }^{35}$. Il est alors nécessaire de réinscrire clairement le harcèlement sexuel au travail dans la dimension du milieu de travail.

Historiquement, avec la venue de l'industrialisation et du capitalisme, on a scindé la possession du capital et du travail. Le contrôle qui appartenait totalement à l'artisan au début du XIX $\mathrm{X}^{\mathrm{e}}$ siècle passe en très grande partie dans les mains du détenteur de capital au cours de $\mathrm{XX}^{\mathrm{e}}$ siècle. Ce contrôle sera de plus en plus exercé par des intermédiaires (la gestion, du contremaître au directeur), le travail en grande série ne favorisant pas l'implication constante du détenteur du capital. «La division du travail introduit ain-

31 Vincent DE FRANCIS, Protecting the Child Victim of Sex Crimes Committed by Adults, 1969, 230 pp.; Michelle R. MAROIS, Camille MESSIER et Louise A. PERREAULT, L'inceste: une histoire à trois et plus, $1982,171 \mathrm{pp}$.

32 Julia TURNER and Ian AUSTIN, «Professors Told to Keep Students at Arm's Lenght», The Globe and Mail, March 10, 1980, pp. 1-2; Donna J. BENSON and Gregg E. THOMPSON, "Sexual Harassment on a University Campus: The Influence of Authority Relations, Sexual Interest and Gender Stratification", Social Problems, Vol. 29, No. 3, Fév. 1982, pp. 236-251; «Sexual Harassment: A Hidden Issue», Project on the Status and Education of Women, June 1978, 7 pp.

33 Au Canada, un viol a lieu à toutes les dix-sept minutes, LAURIN et VOGHEL, op. cit., p. 212.

34 Célyne LACERTE-LAMONTAGNE et Yves LAMONTAGNE, Le Viol: acte de pouvoir et de colère, 1980, p. 13.

35 Dans les diverses études qui se sont intéressées à des répondants des deux sexes, on peut constater un rapport de 3 à 4 femmes identifiant un vécu de harcèlement sexuel au travail pour un homme. De plus, une très grande majorité des harceleurs étaient des hommes quelque soit le sexe de la personne harcelée. "Federal Cases (Report of the Merit Systems Protection Board in Sexual Harassment in the Federal Workforce)", Accross the Board, Vol. 18, No. 9, October 1981, pp. 2-5; Commission canadienne des droits de la personne, op. cit., pp. 7-8; Bryan GOULD and Linda MACDOUGALL, «Keeping Women Down», New Statesmen, Vol. 102, October 23, 1981, pp. 13-14. 
si et justifie une hiérarchie ${ }^{36} . »$ On crée ainsi un nouveau groupe d'individus qui sont investis dans le lieu de travail de beaucoup de droits, de pouvoir. Cette reconnaissance sociale de ce pouvoir est claire et est traduite, en matière de relations de travail, par exemple, par la notion des droits résiduaires de la gérance, ${ }^{37}$ ou par la règle «obey now, grieve later ${ }^{38}$.

Il y a donc dans un milieu de travail, quelqu'il soit, une structure hiérarchique qui investit les supérieurs de nombreux pouvoirs et les subordonnés de nombreux devoirs. Cette structure sociale interne à toute entreprise contient le potentiel nécessaire à faire surgir les abus de pouvoir.

Ces abus peuvent prendre diverses formes dont le harcèlement quotidien dans le travail qui pourrait être le fait tant de harceleurs masculins que féminins. Le harcèlement sexuel au travail pourrait donc s'inscrire comme une des dimensions du harcèlement dans le travail, la dimension sexualité s'y ajoutant et pouvant être plus généralement le fait de harceleurs masculins.

En effet, il est évidemment plus que probable que les perceptions et les comportements des hommes et des femmes face à la sexualité et aux rôles sexuels trouvent aussi le chemin de l'usine ou du bureau en s'exprimant de diverses façons «déviantes», soit selon certains auteurs, par les comportements amoureux ou harcelants ${ }^{39}$.

Le fait que de nombreuses personnes, tant de sexe masculin que de sexe féminin, croient beaucoup plus à l'existence de femmes utilisant leur sexualité pour obtenir des avantages dans leur milieu de travail qu'à l'existence du harcèlement sexuel au travail, ${ }^{40}$ en empêchent encore plusieurs d'effectuer une distinction entre les deux types de comportements déviants. Une telle

36 M. de MONTMOLLIN, Le taylorisme à visage humain, 1981, p. 11.

37 Par cette règle, tout ce qui n'est pas prévu dans une convention collective, est laissé à la discrétion de l'employeur, dans les limites des autres lois de notre pays; voir C. D'AOUST, L. LECLERC et G. TRUDEAU, Les mesures disciplinaires: étude jurisprudentielle et doctrinale, 1982, pp. 62-66; F. MORIN et R. BLOUIN, Précis de l'arbitrage des griefs, 1980, pp. 338-340.

38 Par cette règle, un salarié ne peut refuser d'exécuter un ordre de l'employeur sauf en certaines circonstances bien précises sous peine d'être passible de mesures disciplinaires pour insubordination. Voir à ce propos, C. D'AOUST et G. TRUDEAU, L'obligation d'obéir et ses limites dans la jurisprudence arbitrale québécoise, 1979, $62 \mathrm{pp}$.

39 MEYER et al., op. cit., pp. 109-146.

40 Plusieurs croient même que toutes les femmes ayant atteint un certain niveau hiérarchique dans une entreprise y sont grâce à l'utilisation de leurs charmes. Les lecteurs intéressés par l'analyse des mythes entourant l'utilisation de leur sexualité par les femmes au travail peuvent lire les ouvrages suivants: Robert E. QUINN, «Coping with Cupid: The Formation, Impact and Management of Romantic Relationships in Organizations", in: Neugarten and Shafritz (editors), op. cit., pp. 38-52; BACHOUSE and COHEN, op. cit., pp. 149-156. 
distinction est cependant simple: le comportement amoureux existe lorsqu'il y a réciprocité des sentiments entre les deux partenaires. Le comportement harcelant existe lorsqu'il n'y a pas réciprocité entre les personnes impliquées (la réciprocité impliquant la notion de libre consentement) ${ }^{41}$.

De plus, il semble bien que selon de nombreux auteurs, sous la forme du harcèlement sexuel au travail, se cache principalement l'utilisation que les hommes font de la sexualité dans l'exercice de leurs pouvoirs qu'ils soient économiques, psychologiques ou physiques. Les femmes sont donc abaissées, même au travail, au rang d'objet sexuel comme elles le sont dans la vie de tous les jours ${ }^{42}$. À ce propos, deux auteurs résument bien les attitudes face aux rôles sexuels que les deux sexes intègrent dans leur milieu de travail:

«It arises, at least in part, from a social context in which men have traditionnaly exerted power over women both at home and at work and in which women have been viewed as subordinates. Men have been the sexual initiators, and women, the sexual objects... This pattern has grown from the tradition of women's dependence on men for their material survival, and it has led to the condition in which women, in large part, have been socialized to assess their personnal worth in terms of their desirability to men... and in accepting this pattern, both sexes have legitimized male sexual dominance in the workplace as well as in the society at large. From this perspective sexual harassment of women in the workplace may be viewed as a social mechanism which preserves the status quo, one which reflects more than sexuality itself, the issue of power. $)^{43}$

Pourquoi alors ne pas conclure dans le même sens qu'une étude concernant les motivations des violeurs, ${ }^{44}$ qui souligne que les hommes qui font du harcèlement sexuel au travail, ne recherchent finalement pas une gratification sexuelle mais plutôt la satisfaction que représente l'exercice du pouvoir?

41 Une telle distinction peut être plus difficile à établir lorsqu'un comportement amoureux dégénère en harcèlement sexuel parce que l'un des partenaires désire cesser la relation amoureuse et que l'autre désire la maintenir. L'auteur suivant discute brièvement de ce problème: MEYER et al., op. cit., p. 112.

42 Shelby WHITE, «The Office Pass», Accross the Board, Vol. XIV, No. 4, April 1977, p. 18; LÉVESQUE, op. cit., p. 31; Ginette BOYER, «La femme-objet au travail», Relations, Avril 1982, pp. 102-103; FARLEY, op. cit., p. 31; DAGILIS et NOVAK, op. cit., p. 6; KORDA, op. cit., p. 86; «Mac-Bain: \$1500. d'amende pour harcèlement sexuel», Le Droit, 2 août 1984, p. 5; dans ce dernier cas, un député fédéral a été récemment condamné pour harcèlement sexuel envers une collaboratrice et le tribunal souligne que «... c'est le genre de conduite d'un employeur qui considère son personnel féminin comme des objets ou des ornements sexuels qu'il peut traiter cavalièrement».

43 NEUGARTEN and SHAFRITZ (editors), op. cit., pp. 2-3.

44 LACERTE-LAMONTAGNE et LAMONTAGNE, op. cit., p. 13. 


\section{Définition conceptuelle du harcèlement sexuel au travail}

Cette revue de littérature nous permet d'identifier les composantes de la définition du harcèlement sexuel au travail. Sur le plan conceptuel, c'est la notion de pouvoir et les abus en découlant qui doivent diriger l'emphase d'une définition du harcèlement sexuel en milieu de travail à cause de la structure même des entreprises. Cependant, l'analyse féministe ajoute à la notion de pouvoir hiérarchique la notion du pouvoir social des hommes sur les femmes, particulièrement en matière sexuelle. Les femmes (principalement mais non exclusivement) sont aussi, dans leur milieu de travail, des objets sexuels. Elle sont alors discriminées puisqu'on ne les considère pas uniquement comme travailleuses mais aussi comme femmes sexuées devant subir les avances des hommes.

À la lumière de ces considérations, nous proposons que le harcèlement sexuel au travail soit défini comme suit:

Le harcèlement sexuel au travail est un comportement à connotation sexuelle qui est une manifestation de pouvoir faisant d'une personne un object sexuel dans son milieu de travail.

\section{DÉFINITION OPÉRATIONNELLE}

La définition conceptuelle du harcèlement sexuel au travail retient trois concepts principaux: le concept de pouvoir qui se traduit par le harcèlement, celui de sexualité et celui de milieu de travail. Le Tableau 1 résume les divers critères retenus en regard de ces concepts.

\section{Tableau 1}

Critères permettant de définir opérationnellement le harcèlement sexuel au travail selon les concepts de pouvoir, de sexualité et de milieu de travail

Concepts

Pouvoir

(Harcèlement)

Sexualité

Milieu de travail
Critères

1) les comportements ont pour but ou effet de contrarier ou contraindre une personne;

2) les comportements sont répétés sauf:

a) lorsque l'effet est clairement contraignant;

b) lorsque plusieurs personnes posent le même geste;

c) lorsque des représailles suivent;

3) les comportements sont non réciproques;

4) les comportements ont une connotation sexuelle;

5) le harceleur est une personne reliée au milieu de travail de la victime. 
En ce qui concerne le concept de sexualité, lorsque l'on analyse les divers sondages ou études traitant du sujet, on constate que les comportements harcelants peuvent prendre trois formes, soit non verbale, verbale ou physique ${ }^{45}$.

En ce qui concerne le concept de pouvoir, les comportements se situent aussi sur un continuum allant d'un degré contrariant à un degré contraignant tel que Hughes et $\mathrm{May}^{46}$ l'ont souligné dans leur définition du harcèlement. Cependant, selon plusieurs auteurs, certains comportements tels l'assaut, la tentative de viol ou le viol sont d'un ordre de violence si poussé que l'on peut parler d'un degré agressant ${ }^{47}$.

Il faut préciser que la non réciprocité des comportements est implicitement reconnue par le degré des comportements, la contrariété, la contrainte ou l'agression s'identifiant clairement à des sentiments non réciproques.

Devant nuancer le critère de répétition des comportements observés, la première nuance est établie en fonction du degré atteint par ces comportements. En effet, au niveau des comportements dits agressants, la répétition n'est pas nécessaire pour «déterminer correctement la nature» de ces comportements ${ }^{48}$.

La seconde nuance est établie en fonction de la forme du comportement. Un simple toucher clairement défini (i.e. non pas des frôlements dont on ne peut être certains dès la première occasion s'ils sont accidentels ou pas) peut être une invasion très grande de l'espace vital d'une personne. En effet, plusieurs auteurs soulignent que l'espace vital d'une personne en relation avec une autre, entre autres dans le milieu de travail, serait environ de quatre (4) à douze (12) pieds. Ils situent un espace vital de moins de quatre (4) pieds comme étant celui correspondant à des relations personnelles ou intimes ${ }^{49}$. Les divers comportements de forme physique et de degré contraignant ne devraient donc pas nécessiter de répétition et être définis comme étant du harcèlement sexuel au travail dès leur apparition.

45 Jennifer JAMES, op. cit., p. 404. Notons que cette distinction est presque constante dans toutes les études qui traitent de ce sujet.

46 HUGHES and MAY, op. cit., p. 250.

47 BILES, op. cit., pp. 49-56; pour une élaboration sur cette notion de continuum, voir aussi les ouvrages suivants: Caroll M. BRODSKY, The Harassed Worker, 1976, p. 2; «Love in the Office», Management Review, Vol. 68, No. 11, November 1979, p. 5.

48 DAGILIS et NOVAK, op. cit., p. 11.

49 Ces auteurs soulignent l'utilisation de ce type de communication non verbale dans un contexte de relations de pouvoir. Les lecteurs intéressés par cet aspect peuvent lire les ouvrages suivants: Nancy M. HENLEY, Body Politics: Power, Sex and Non Verbal Communication, 1977, 214 pp.; Michael ARGYLE, Bodily Communication, 1975, 403 pp.; Michael ARGYLE and Mark COOK, Gaze and Mutual Gaze, 1976, 210 pp. 
La troisième nuance est établie en fonction du statut du ou des harceleurs. En effet, une victime peut subir divers comportements qui sont le fait d'une seule personne ou d'un groupe de personnes agissant de concert parmi lesquelles il est souvent possible de déterminer un leader. D'un autre côté, une victime peut subir divers comportements qui sont le fait de plusieurs personnes, non reliées entre elles, chacune d'elles n'agissant qu'une seule fois. Mais, comme certains auteurs l'ont souligné, la répétition des comportements qui identifie le harcèlement ne doit pas nécessairement être effectuée par la même personne ${ }^{50}$.

Enfin, la dernière nuance est établie en fonction des représailles que peut exercer le harceleur. En effet, lorsqu'un comportement à connotation sexuelle n'est pas répété mais est suivi, suite à un rejet de la victime, de représailles, il faut conclure que le critère de répétition du comportement à connotation sexuelle n'est pas alors nécessaire pour identifier le comportement comme étant du harcèlement sexuel au travail.

Il faut aussi souligner que les divers comportements à connotation sexuelle qu'une victime peut subir peuvent avoir lieu autant en milieu de travail qu'en dehors du milieu du travail, le lien avec le milieu de travail étant alors assuré par le statut du harceleur. Il est alors possible de définir opérationnellement le harcèlement sexuel au travail comme étant:

tout comportement à connotation sexuelle, généralement répétitif, non réciproque, dont le but ou l'effet est de contrarier ou de contraindre un(e) travailleuse-eur à faire quelque chose qu'elle(il) n'aurait pas fait sans cette contrainte.

Enfin, il est possible d'établir un cadre opérationnel en identifiant les divers comportements à connotation sexuelle qui peuvent être considérés comme étant du harcèlement sexuel au travail. Le Tableau 2 reproduit ce cadre opérationnel en identifiant pour chacune des formes et degrés retenus les divers comportements à connotation sexuelle possibles ${ }^{51}$.

\section{CONCLUSION}

Le harcèlement sexuel au travail s'inscrit clairement dans le cadre des relations de pouvoir issues de la structure des milieux de travail auquel s'ajoute la dimension des relations de pouvoir entre les hommes et les femmes dans toutes les sphères de la société y compris en milieu de travail.

50 HUGHES and MAY, op. cit., p. 251.

51 Cette revue des types de comportements harcelants a été effectuée à partir de l'ensemble de la littérature sur le sujet, à l'aide de questionnaires déjà construits et d'histoires de cas. 


\section{Tableau 2}

Les formes et degrés du harcèlement sexuel au travail: cadre opérationnel

\begin{tabular}{|c|c|c|c|}
\hline Degré $\quad$ Forme & Non verbale & Verbale & Physique \\
\hline Contrariant & $\begin{array}{l}\text { * regards, } \\
\text { * sifflements, } \\
\text { * photos, textes, etc. }\end{array}$ & $\begin{array}{l}\text { * blagues, remarques, } \\
\text { * poser questions } \\
\text { intimes, etc. }\end{array}$ & $\begin{array}{l}\text { * frôlement, } \\
\text { * tapotements, } \\
\text { etc. }\end{array}$ \\
\hline Contraignant & $\begin{array}{l}\text { * petits présents, } \\
\text { * flâner devant son } \\
\text { domicile ou lieu } \\
\text { de travail }\end{array}$ & $\begin{array}{l}\text { * demandes de sorties } \\
\text { * offres concernant } \\
\text { travail } \\
\text { * offres vie hors } \\
\text { travail, etc. }\end{array}$ & $\begin{array}{l}\text { - caresse, } \\
\text { - embrasser } \\
\text { - pincer, } \\
\text { - empoigner, } \\
\text { - soulever } \\
\text { vêtement, } \\
\text { - acculer dans } \\
\text { un coin, etc. }\end{array}$ \\
\hline Agressant & $\begin{array}{l}\text { - lettres de menaces } \\
\text { - aller au domicile } \\
\text { - suivre la personne } \\
\text { harcelée } \\
\text { - exhibitionnisme, } \\
\text { etc. }\end{array}$ & $\begin{array}{l}\text { - téléphones obscènes, } \\
\text { anonymes, de } \\
\text { menaces } \\
\text { - insinuation à autrui } \\
\text { - menaces concernant } \\
\text { travail, } \\
\text { - propositions, } \\
\text { sexuelles } \\
\text { - refus d'accepter fin } \\
\text { des relations } \\
\text { amoureuses, etc. }\end{array}$ & $\begin{array}{l}\text { - arracher } \\
\text { vêtement, } \\
\text { assaut, } \\
\text { tentative de } \\
\text { viol, } \\
\text { - viol }\end{array}$ \\
\hline
\end{tabular}

* Les comportements doivent être répétés sauf si des représailles s'ensuivent.

Cependant, parce que tant le sujet des relations de pouvoir que celui de la sexualité sont délicats, il est nécessaire de définir concrètement le harcèlement sexuel au travail en apportant de nombreuses nuances. Il n'en demeure pas moins que la perception d'une situation donnée comme étant du harcèlement sexuel sera toujours celle de la personne la subissant. C'est pourquoi des critères tels que le non consentement (implicite ou explicite) et la répétition des comportements viennent nuancer l'analyse qu'une personne harcelée peut se faire de sa situation et permettre au présumé harceleur de prendre conscience de l'inconvenance de certaines actions qu'il peut poser, dans certains cas, «en toute bonne foi».

Il n'en demeure pas moins que certains des comportements acceptables ou même valorisés autrefois sont aujourd'hui rejetés par l'ensemble des femmes, qu'elles se considèrent ou non féministes. Ces changements de per- 
ceptions doivent aussi trouver leur contre partie chez les hommes parce que les femmes sont dans les milieux de travail pour y rester et pour s'y implanter de plus en plus envers et contre, entre autres, le harcèlement sexuel au travail.

\section{BIBLIOGRAPHIE}

ARGYLE, Michael, Bodily Communication, London, Methuen, 1975, 403 pp. ARGYLE, Michael and Mark COOK, Gaze and Mutual Gaze, Cambridge, Cambridge University Press, 1976, $210 \mathrm{pp}$.

ARMSTRONG, Pat and Hugh ARMSTRONG, $A$ Working Majority: What Women Must Do for Pay, Ottawa, Canadian Advisory Council on the Status of Women, 1983, $280 \mathrm{pp}$.

BACHOUSE, Connie et al., Fighting Sexual Harassment: An Advisory Handbook, Cambridge, Mass., Alliance Against Sexual Harassment, 1979, 92 pp.

BACHOUSE, Constance and Leah COHEN, Sexual Harassment on the Job: How to Avoid Working Woman's Nightmare, Toronto, MacMillan of Canada, 1981, $196 \mathrm{pp}$.

BAYLES, Michael, "Coercive Offers and Public Benefits», The Personalist, Vol. 55, spring 1974, pp. 142-143.

Bell and Korczak v. Ernest Ladas and the Flaming Steer Steak House, 1 C.H.R.R. D/155, le 12 août 1980 , O.B. Shime.

BELOTTI, Elena G., Du côté des petites filles (seconde édition), Paris, Éditions des femmes, 1974, $251 \mathrm{pp}$.

BENSON, Donna J. and Gregg E. THOMPSON, «Sexual Harassment on a University Campus: The Influence of Authority Relations, Sexual Interest and Gender Stratification», Social Problems, Vol. 29, No. 3, Fév. 1982, pp. 236-251.

BILES, Georges E., «A Program Guide for Preventing Sexual Harassment in the Workplace», The Personnel Administrator, Vol. 26, No. 6, June 1981, pp. 49-56.

BOYER, Ginette, «La femme-objet au travail», Relations, Vol. 42, No 479, Avril 1982, pp. 102-103.

BRADFORD, David L. et al., "The Executive Man and Woman: The Issue of Sexuality", in Neugarten and Shafritz (Editors), Sexuality in Organizations: Romantic and Coercive Behaviors at Work, Oak Park, III, Moore 1980, $166 \mathrm{pp}$.

BRODSKY, Caroll M., The Harassed Worker, Lexington, Mass., Heath, 1976, $174 \mathrm{pp}$.

BRODSKY, Caroll M., «Rape at Work», in J. Marcia Walker and Stanley L. Brodsky (editors), Sexual Assault: The Victim and The Rapist, Mass., Lexington, 1976, pp. 35-51. 
BROWNMILlER, Susan, Le viol, Paris, Montréal, Stock-L'Étincelle, 1976, $569 \mathrm{pp}$.

Charte des droits et libertés de la personne, L.R.Q., c. C-12.

COLLINS, Eliza G.C. and Thimothy B. BLODGETT, «Sexual Harassment... Some See It... Some Won't», Harvard Business Review, Vol. 59, No. 2, March-April 1981, pp. 76-95.

COMITÉ D'ACTION SUR LE HARCÈLEMENT SEXUEL, «Questionnaire sur le harcèlement sexuel», $A$ u Bas de l'Échelle, Circa, 1982, 6 pp. (non publié).

COMMISSION CANADIENNE DES DROITS DE LA PERSONNE, Attentions sexuelles non sollicitées et harcèlement sexuel: résultats d'un sondage auprès des Canadiens, Ottawa, ministère des Approvisionnements et Services, Canada, 1983, 28 pp.

CRULL, Peggy, «The Impact of Sexual Harassment on the Job: A Profile of the Experiences of 92 Women», in Neugarten, Dail Ann and Jay M. Shafritz (Editors), Sexuality in Organizations: Romantic and Coercive Behaviors at Work, Oak Park, Illinois, Moore 1980, $166 \mathrm{pp}$.

DAGILIS, André J. et Elisabeth NOVAK, Le harcèlement sexuel? Connais pas..., Circa, 1982, 40 pp. (non publié).

D'AOUST, Claude, Louis LECLERC et Gilles TRUDEAU, Les mesures disciplinaires: étude jurisprudentielle et doctrinale, Montréal, École de relations industrielles de l'Université de Montréal, 1982, 484 pp.

D'AOUST Claude et Gilles TRUDEAU, L'obligation d'obéir et ses limites dans la jurisprudence arbitrale québécoise, Montréal, École de relations industrielles de l'Université de Montréal, 1979, 62 pp.

DE FRANCIS, Vincent, Protecting the Child Victim of Sex Crimes Committed by Adults, Denver, American Human Association, Children's Division, 1969, 230 pp.

DE MONTMOLLIN, M., Le taylorisme à visage humain, Paris, P.U.F., 1981, 167 pp.

janvier-février 1982, pp. 35-38.

DRISCOLL, Jeanne Bosson, «Sexual Attraction and Harassment: Management's New Problems», Personnel Journal, Vol. 60, No. 1, January 1981, pp. 33-36, 56.

DRISCOLL, Jeanne Bosson and Rosemary A. BOVA, «The Sexual Side of Enterprise», Management Review, Vol. 69, No. 7, July 1980, pp. 51-54.

FARLEY, Lin, Sexual Shakedown: The Sexual Harassment of Women on the Job, New-York, Warner, 1978, 288 pp.

_____ «Federal Cases (Report of the Merit Systems Protection Board on Sexual Harassment in the Federal Workforce)», Accross the Board, Vol. 18, No. 9, October 1981 , pp. 2-5.

FÉDÉRATION DES TRAVAILLEURS DU QUÉBEC, Le harcèlement sexuel, C'est quoi?, Montréal, F.T.Q., 1983, 48 pp. 
GOULD, Bryan and Linda MACDOUGALL, «Keeping Women Down», New Statesmen, Vol. 102, October 23, 1981, pp. 13-14.

HENLEY, Nancy M., Body Politics: Power, Sex and Non Verbal Communication, Englewood Cliffs, New-Jersey, Prentice-Hall, 1977, 214 pp.

HIVELY, Janet M. and William S. HOWELL, «The Male-Female Management Team: A Dance of Death?», Management Review, Vol. 69, No. 6, June 1980, pp. 44-50.

HUGHES, John C. and Larry MAY, "Sexual Harassment», Social Theory and Practice, Vol. 6, No. 3, Fall 1980, pp. 249-280.

JAMES, Jennifer, «Sexual Harassment», Public Personnel Management, No. 4, Winter 1981, pp. 402-407.

KLEMMACK, Susan H. and David L. KLEMMACK, "The Social Definition of Rape», in J. Marcia Walker and Stanley L. Brodsky (editors), Sexual Assault: The Victim and The Rapist, Mass., Lexington, 1976, pp. 135-147.

KORDA, Michaels, Male Chauvinism' How it Works, New-York, Random House, 1972, 244 pp.

Kotyk and Allary v. Canadian Employment and Immigration Commission and Jack Chuba, 4 C.H.R.R. D/1416, le 20 avril 1983, Susan M. Ashley.

LACERTE-LAMONTAGNE, Célyne et Yves LAMONTAGNE, Le viol: acte de pouvoir et de colère, Montréal, La Presse, 1980, 132 pp.

LAURIN, Lucie et Johanne VOGHEL, Viol et brutalité, Montréal, QuébecAmérique, 1983, $264 \mathrm{pp}$.

LÉVESQUE, Mariette, Bas les pattes! Le harcèlement sexuel, Montréal, Québécor, $1982,252 \mathrm{pp}$.

tawa, 2 août 1984, p. 5.

Loi canadienne sur les droits de la personne, S.C. 1976-1977, c. 33.

1979 , p. 5.

MAC KINNON, Catherine A., Sexual Harassment of Working Women: A Case of Sex Discrimination, New-Haven, Yale University Press, 1979, 312 pp.

MAROIS, Michelle R., Camille MESSIER et Louise A. PERREAULT, L'inceste: une histoire à trois et plus, Québec, Comité de la protection de la jeunesse, 1982, $171 \mathrm{pp}$.

MEAD, Margaret, Male and Female, New-York, Morrow, 1949, 477 pp.

_-___, Moeurs et sexualité en Océanie, Paris, Plon, 1971, 533 pp.

____ Sex and Temperaments in Three Primitives Societies, (third edition), New-York, Morrow, 1963, 335 pp.

_____, "A Proposal: We Need Taboos on Sex at Work», in Neugarten and Shafritz (editors), Sexuality in Organisations, Romantic and Coercive Behaviors at Work, Oak Park, Ill., Moore 1980, 166 pp. 
MEYER, Mary Coeli et al., Sexual Harassment, Princeton, New-York, Petrocelli, $1981,200 \mathrm{pp}$.

MOISAN, Lise, «Les dessous du 9 à 5: suite et fin?», La Vie en Rose, Montréal, septembre-octobre 1982, pp. 16-18, 70-71.

MONTGOMERY, Charlotte, «Ottawa Bans Harassment on the Job», Globe and Mail, Toronto, December 30, 1982, pp. 1-2.

MORIN, Fernand et Rodrigue BLOUIN, Précis de l'arbitrage des griefs, Québec, P.U.L., 1980, 507 pp.

MURPHY, Robert F., «Social Structure and Sex Antagonism», Southwestern Journal of Anthropology, Vol. 15, 1959, pp. 89-98.

NEUGARTEN, Dail Ann and Jay M. SHAFRITZ (editors), Sexuality in Organizations: Romantic and Coercive Behaviors at Work, Oak Park, Illinois, Moore, 1980, $166 \mathrm{pp}$.

OLIVIER, Christiane, Les enfants de Jocaste, Paris, Denoël-Gonthier, 1980, $192 \mathrm{pp}$.

QUINN, Robert E. and Noreen A. JUDGE, «How to Cope with Cupid... the Office Romance: No Bliss for the boss", Management Review, Vol. 67, No. 7, July 1978, pp. 43-49.

QUINN, Robert E., «Coping with Cupid: The Formation, Impact and Management of Romantic Relationships in Organizations», in Neugarten and Shafritz (Editors), Sexuality in Organizations: Romantic and Coercive Behaviors at Work, Oak Park Ill., Moore 1980, 166 pp.

RENICK, James C., «Sexual Harassment at Work: Why it Happens, What to Do About It», Personnel Journal. Vol. 59, No. 8, August 1980, pp. 658-662.

ROGERS, Madeline, «Is the Office Affaires Worth It?», MBA/-Masters in Business Administration, Vol. 12, No. 2, February 1978, pp. 65-66.

ROSE, Herbert J., A Handbook of Greek Mythology, New-York, Dutton, 1928, 363 pp.

SAFRAN, Claire, «Sexual Harassment: The View From the Top», Redbook, March 1981, pp. 45-51.

- _ - - «Sexual Harassment: A Hidden Issue», Project on the Status and Education of Women, June 1978, 7 pp.

____—, «Solidarity Wins», Ms. September 1980, p. 85.

TARNOPOLSKY, Walter Surma, Discrimination and the Law in Canada, Toronto, Richard De Boo, 1982, 595 pp.

THURSTON, Kathryn A., «Sexual Harassment: an Organizational Perspective», The Personnel Administrator, Vol. 25, No. 12, December 1980, pp. 59-64.

TURNER, Julia and Ian AUSTIN, «Professors Told to Keep Students At Arm's Lenght», The Globe and Mail, Toronto, March 10, 1980, pp. 1-2.

WHITE, Shelby, «The Office Pass», Across the Board, Vol. XIV, No. 4, April 1977, pp. 17-20. 


\section{Sexual Harassment at Work Definition and Measurement}

The first part of this study investigated the different definitions of sexual harassment at work. The authors also proposed a theoretical and operational definition of this complex concept. The second part of the study suggested an operational framework integrating not only the different forms but also the degrees under which this phenomenon materialised.

A review of the literature identified two dominant trends: the legalistic school with a mainly descriptive definition and the feminist school more interested in the balance of power between men and women. It is this notion of power and the resulting abuses that should in effect predominate in a definition of sexual harassment at work.

Nevertheless, the feminist analysis alone cannot define the total phenomenon even though the most common relationship is that of a woman-harassee and a manharasser. A subsequent analysis of power in the workplace brings out the constant presence of an hierarchy which vests superiors with many prerogatives. This internal social structure in any enterprise potentially leads to abuses of power. Sexual harassment at work may therefore be categorized as one of the dimensions of harassment at work, the sexual dimension encompassing the relation of power between men and women found at the heart of feminist analysis. This analysis adds to the notion of hierarchical power, that of social domination of women by men, particularly in sexual matters. Women are thus discriminated against since they are not considered solely as workers but also as sexual females having to be subjected to male advances.

Sexual harassment at work may thus be defined as follows: a behaviour of a sexual connotation as a manifestation of power making a person a sexual object in his/her workplace.

The operational definition must take into account three important dimensions: power (operative in function of the degree of the behaviour reached: annoyance, coercion or assault), sexuality (three forms of behaviour: non verbal, verbal or physical) and the workplace (the harasser having a tie to the workplace of the harassee though the harassment mayor may not occur in the workplace). The literature retains two elements essential to an operational definition: the notion of non-consent or non-reciprocity (clearly indicated by the degree of the behaviour) and that of repeatedness. This last element must be qualified, as legal experts and theorists have done, in function of various criteria. For example, as regards the degree of behaviour, it is obvious that an assault does not have to be repeated «in order to determine correctly its nature».

Sexual harassment at work may therefore be defined operationally as being any behaviour of sexual connotation, generally repetitive, non-reciprocal, with the object or the effect of annoying or coercing a worker to do something that he/she would not otherwise do. 
In order to present an operational framework enabling systematic research on the presence of such behaviour, it is possible to situate each type of behaviour in function of the form and the degree reached, and this is what is show in Table 2.

This exercice in definition provides for the study of the characteristics of the problem as experienced in a firm or in the whole of society. It also situates very well sexual harassment within the framework of relations of power stemming from the structure of workplaces to which is added the balance of power between men and women in all spheres of society, the workplace included. Because women are in the workplace to stay and to take their place against all odds amongst which sexual harassment, it is obvious that changes in perceptions and attitudes are expected of men.

Pour mieux saisir les problèmes du travail et les questions sociales dans le monde, lisez la

\section{REVUE INTERNATIONALE DU TRAVAIL}

\section{Quelques articles récents:}

- Les programmes spéciaux d'emploi dans les pays de R.G. Hollister, Jr. I'OCDE et David $\mathrm{H}$. Freedman

- La participation des travailleurs et les politiques du personnel en Israël

A. Bar-Haïm

- Décisions judiciaires en matière de droit du travail

- Les syndicats et les médias

G. Walsh

- La participation financière des travailleurs, un moyen de vaincre le chômage?

G. Standing

La Revue internationale du Travail est aussi publiée en anglais. Abonnement annuel (1988): SF 60.00; Can. $\$ 54$

Bureau International du Travail, Publications, $\mathrm{CH}-1211$. Genève 22, Suisse Succursale canadienne du BIT, Bureau 202, 75, rue Albert, Ottawa (Ont.) K1P 5E7, Tél.: (613) 233-1114 